Research Square
Preprints are preliminary reports that have not undergone peer review.

They should not be considered conclusive, used to inform clinical practice, or referenced by the media as validated information.

\title{
Determining The Age Priority in Universal Vaccination Using Economic Evidence: Case From US Based On Dynamic Transmission Model
}

\author{
Dachuang Zhou \\ China Pharmaceutical University \\ Taihang Shao \\ China Pharmaceutical University \\ Zeyao Liu \\ China Pharmaceutical University \\ Xingming Pan \\ China Pharmaceutical University \\ Xueke Zhang \\ China Pharmaceutical University \\ Chengchao Zhou \\ Shandong University \\ Wenxi Tang ( $\sim$ tokammy@cpu.edu.cn ) \\ China Pharmaceutical University
}

\section{Research Article}

Keywords: COVID-19, vaccine, dynamic transmission model, allocation strategy, cost-effectiveness analysis

Posted Date: August 18th, 2021

DOI: https://doi.org/10.21203/rs.3.rs-789689/v1

License: (c) (i) This work is licensed under a Creative Commons Attribution 4.0 International License. Read Full License 


\section{Abstract}

Background: Currently, increasing regions have realized that universal vaccination are necessary to prevent COVID-19. However many of them are facing problems associated with insufficient supply or chaotic allocation of vaccines. This study selected the United States population as an example and explored prioritization strategies of COVID-19 vaccination for different age groups to achieve the highest economic efficiency.

Methods: We built a dynamic transmission model to predict the incidence of SARS-CoV-2 infections under the prioritization strategies of vaccination for different ages within a 180-day simulation period. Quality-adjusted life year (QALY) was selected as the outcome. Medical costs included direct medical cost and vaccine cost based on a healthcare system perspective. Data on SARS-CoV-2 epidemiology, vaccine efficacy, and medical costs were derived from publicly available databases and previously published literature. Different COVID-19 vaccines were included in scenario analysis. The robustness of the study results was evaluated by one-way sensitivity analysis and probabilistic sensitivity analysis.

Results: COVID-19 vaccination is economical compared with no vaccination. Priority vaccination for adults aged $25-59$ years saves $\$ 31,664.2$ million and that for adults over 60 years old saves $\$ 30,082.9$ million in medical costs compared with no vaccine intervention. Additionally, priority vaccination for adults aged 25-59 years vs. over 60 years old saves $\$ 1,581.3$ million. In contrast, priority vaccination for adults aged over 60 years vs. 25-59 years old gains 0.001 QALYs and costs $\$ 4.7$ per capita, with an incremental cost-effectiveness ratio of $\$ 4,829.3 /$ QALY, and it is economical when taking gross domestic product per capita of the United States in 2020 as the willingness-to-pay threshold. The results of sensitivity analysis indicate that the base-case results are robust.

Conclusions: From a healthcare system perspective, it is most economical to prioritize adults aged over 60 years for COVID-19 vaccination in the United States, thereby achieving effective resource allocation and saving the government costs.

\section{Background}

The severe acute respiratory syndrome caused by the novel coronavirus (SARS-CoV-2, previously 2019-nCoV), which first emerged at the end of 2019, spread rapidly around the world and reached 58 countries/regions by March 1,2020[1]. As of April 20, 2021, a total of 141,549,845 confirmed cases of coronavirus disease 2019 (COVID-19) were reported globally, with 3,021,397 deaths. Of these, 31,350,025 cases were confirmed in the United States, with 561,921 deaths [2]. SARS-CoV-2, which is characterized by high infectivity and low lethality, differs from the previously prevalent coronaviruses SARS-CoV and MERS-CoV. The proven transmission routes of SARS-CoV-2 include transmission via droplets, close contact, respiratory mucosa, and contact with virus-contaminated items [3]. The incubation period after SARS-CoV-2 infection usually lasts for 1-14 days, with a mean of 5.3 days. Most patients develop clinical symptoms 3-7 days after infection $[4,5]$. The mean reproductive rate (R0) of COVID-19 is approximately $2.5[6]$, with a mean mortality rate of $2.2 \%$ and cure rate of $\sim 71.8 \%[7$, 8]. The economic burden of COVID-19 is serious. According to a study based on the United States population, the direct medical costs of a single COVID-19 case for the treatment of COVID-19 alone (excluding the treatment of concomitant diseases) reach $\$ 3,045$ US dollars. Furthermore, a study conducted in mainland China showed that the economic burden per COVID-19 patient was \$2,629.9 in China as of March 15, 2020 [9].

Although many therapeutic drugs or regimens have been proven to be effective in the treatment of COVID-19 [10-12], the top priority of COVID-19 control is to prevent SARS-CoV-2 transmission from the source, rather than to find therapeutic drugs suited for the treatment of COVID-19. There are two approaches for a safe and effective vaccine to help protect susceptible people. One is direct protection, namely vaccinating people with high infection risk to prevent disease development, and the other is indirect protection, namely vaccinating people who are in contact with high-risk groups to reduce disease spread. A phase II clinical trial of the world's first vaccine against SARS-CoV-2 reported results from July 2020 [13]. Sixty new COVID-19 coronavirus vaccine candidates had been approved for clinical trials as of December 2020 [14]. In total, 793,484,083 doses of COVID-19 vaccines had been administered worldwide by April 18, 2021 [2]. The emergence of COVID-19 vaccines brought hope to the prevention of diseases and control of costs related to SARS-CoV-2. However, there are still many controversies in terms of vaccine production scale, protection rate, safety, price acceptability, and allocation. Since 2021 , increasing countries have launched the plan of universal vaccination and the United Nations also called for effective vaccines available for all [15]. However, low-income and middleincome countries cannot get or manufacture enough vaccines; high-income countries did not allocate vaccines orderly [16, 17]. Take the United States as an example, the government has promised to provide enough vaccines for all eligible Americans [18], but chaotic vaccination allocation is a public health threat [19]. Disorganized vaccination is equivalent to large-scale crowd gathering, which will cause more unnecessary infections without protective measures. Therefore, in the case of either available or limited vaccine supply, the government's reasonable allocation of resources to realize cost-effectiveness is critical.

Currently, different people have various opinions on who should be prioritized for vaccination against COVID-19. Some people contend that in most cases, vaccines should be prioritized for adults over 60 years old, because the mortality rate is relatively high among people aged over 60 years, and priority vaccination in this age group can minimize the population mortality and years of life lost [20]. One option is to prioritize patients with long-term chronic diseases and people with the greatest occupational risk for vaccination; other people should have equal priority for receiving the vaccine, and it is undesirable to prioritize people aged over 60 years. Another option is to leave $10 \%$ of the total vaccines for people within the lowest quartile of stroke volume index in a federal state, and ensure that special effort is made to provide residents in high-vulnerability areas with vaccines [21]. All these vaccination strategies are supported by persuasive reasoning, yet which program is the most appropriate remains inconclusive.

Evaluating the prioritization of vaccination from an economic perspective provides an appropriate angle for nationwide strategies. Currently, economic evaluations on COVID-19 vaccines are still scarce owing to the short time that COVID-19 vaccines have been on the market and the lack of data related to COVID-19. The objective of this study was to build a dynamic transmission model for COVID-19 based on the United States population using available clinical trial data and epidemiological parameters. Considering that most people in the United States receive the COVID-19 vaccine produced by Pfizer[22], we selected the Pfizer vaccine as the primary research object and explored other scenarios in scenario analysis. The economic evaluation of different vaccination strategies from a healthcare system perspective can provide a reference for the United States and other countries especially the countries that have not carried out COVID-19 vaccination and need to use limited resources effectively.

Page 2/17 


\section{Methods}

\subsection{Objectives}

A dynamic transmission model was used to explore different prioritization orders of vaccination against COVID-19 (priority vaccination for different age groups or no vaccination) for all people in the United States based on an economic perspective, without considering changes in population due to international migration. This research has been registered on PROSPERO and the registration number is CRD 42021240072.

\subsection{Study perspective}

We selected a healthcare system perspective and considered the direct benefits COVID-19 patients get through the health resource consumption caused by vaccination.

\subsection{Participants}

The study included healthy people who had never suffered from COVID-19 throughout the United States. The participants were divided into four age groups: 0-19 years, 20-24 years, 25-59 years, and over 60 years.

\subsection{Intervention and control}

The study compared the economy of vaccination prioritization for different age groups and no vaccination.

Program 1: Priority is given to vaccination for young people at $25-59$ years old, followed by over 60 years, then $20-24$ years, and finally $0-19$ years. A second dose of vaccine is administered after all age groups have received one dose.

Program 2: Priority is given to vaccination for people over 60 years old, followed by $25-59$ years, then $20-24$ years, and finally $0-19$ years. A second dose of vaccine is administered after all age groups have received one dose.

Program 3: No vaccine is administered for intervention.

The vaccine used in the study is BNT162b2 mRNA manufactured by Pfizer. A total of two doses are required. After the first dose is administered, the second dose should be administered 21 days later.

\subsection{Outcomes}

COVID-19 incidence rate was used as a proxy indicator of vaccine efficacy. Additionally, incremental cost-effectiveness ratio (ICER) and net benefit were used as economic decision-making indicators.

\subsection{Model design}

\subsubsection{Dynamic transmission model structure}

The United States population was divided into 10 major health states, and some health states were sub-divided according to age. In the dynamic transmission model, healthy people are infected via contact with people who have been infected with the virus but have not developed the disease, or with exposed people who have developed the disease. Over time, the infection progresses from latent to active, and the infected people choose to receive treatment or not. Although vaccination can reduce the infection risk, the vaccine's protection rate is not $100 \%$. There is still a risk of being infected after vaccination, and vaccinated people remain susceptible in a strict sense. The specific model structure is shown in Figure 1.

Simulation period The study period was set to 6 months for four reasons. (1) Future changes in national policies and individual behaviors across various countries are likely to influence disease progression. Simulation over a too long period of time may result in large differences compared with the real situation of the disease. Taking 6 months as the study period can reduce the uncertainty of the model and improve its authenticity to a certain degree. (2) The supply of vaccines is limited. Although the United States government promises that every American can be vaccinated, a 180-day study period reduces parameter uncertainty by taking into account the actual situation of vaccine production and transportation, vaccination in batches by the day as a unit, and a lack of strong evidence supporting the effective protection period and sequelae of vaccine. (3) Acute infectious diseases are typically characterized by a short duration, with little influence on the cost-effectiveness. Model simulation for too long a period of time therefore results in greater uncertainty. (4) According to existing clinical evidence [23], the immune period for SARS-CoV-2 of people who have recovered after infection is approximately 6 months. If the study period is set to a year, then it is necessary to consider the case of removed people, namely recovered people who have again become susceptible to infection, as well those who have died naturally, which greatly increases the model's complexity and distortion. 
Cycle period The simulation involved 180 one-day cycles. The transfer rate between health states was calculated using a differential equation composed of the number of infected people and the infection rate. The choice of the cycle period was mainly based on the strong infectivity of COVID-19, with relatively large changes during disease progression. If using a longer cycle period, the estimation of indirect protection would be distorted to a greater degree.

\subsubsection{Model Assumption}

COVID-19 is an acute infectious disease with rapid development and multiple routes of infection. COVID-19 vaccines have been on the market for a short time and there are limited data on COVID-19, making it almost impossible to acquire data from all the same samples. Therefore, we made the following assumptions while keeping as close to the real situation as possible:

(1) We assume there is no infection risk for hospitalized people.

(2) Despite the possibility of infection in healthy people who have contacted the virus in nature, there is currently no strong evidence indicating that it is a major source of infection. Therefore, the possibility of infection in healthy people who have contacted the virus in nature or on virus-contaminated items was disregarded in this study.

(3) Although SARS-CoV-2 mutations have occurred, virus mutation per se is a complicated event occurring by chance, and the infection rate after mutation shows greater uncertainty. To ensure the study would be conducted smoothly, we assume that the virus continues to be infectious and will no longer evolve.

(4) We assume that people who have recovered from COVID-19 infection have $100 \%$ immunity in the study period.

(5) The cost of adverse reactions was not taken into account because there have been very few reports on adverse reactions to the COVID-19 vaccine, and considering the reported anaphylaxis in rare cases [24].

(6) No cost was considered for death.

(7) We assuming that there is no age limit for vaccination; that is, all age groups can be vaccinated.

Furthermore, parameters such as mother-to-child transmission and births among the study population were not considered in the study because of insufficient evidence of transmission routes and the relatively short study period.

\subsubsection{Model Parameters}

The baseline values of demographic, epidemiological, vaccine, and economic parameters (Table 1) were determined through extensive searches of published literature and associated data in major databases (e.g., PubMed, Web of Science, and Cochrane Library), some authoritative websites (e.g., World Health Organization (WHO)), and United States Centers for Disease Control and Prevention (CDC). The calculations and sources of key parameters are introduced briefly in the following subsections. 
Table 1

Model inputs

\begin{tabular}{|c|c|c|c|c|c|}
\hline Initial number of susceptible people & Value & Range & & Distribution & Source \\
\hline \multicolumn{6}{|l|}{ Susceptible people } \\
\hline$\geq 60$ & 77047000 & / & / & / & \multirow[t]{4}{*}[25]{} \\
\hline $25-59$ & 153332000 & / & / & I & \\
\hline $20-24$ & 21616000 & l & l & I & \\
\hline $0-19$ & 83520000 & / & / & / & \\
\hline \multicolumn{6}{|c|}{$\begin{array}{l}\text { Healthy people who have received covid-19 } \\
\text { vaccine ( } 1 \text { dose) }\end{array}$} \\
\hline$\geq 60$ & 16198501 & / & / & gamma & \multirow[t]{4}{*}[22]{} \\
\hline $25-59$ & 7391461 & l & / & gamma & \\
\hline $20-24$ & 602902 & / & / & gamma & \\
\hline $0-19$ & 265949 & / & / & gamma & \\
\hline \multicolumn{6}{|c|}{$\begin{array}{l}\text { Healthy people who have received covid-19 } \\
\text { vaccine ( } 2 \text { doses) }\end{array}$} \\
\hline$\geq 60$ & 14399984 & / & / & gamma & \multirow[t]{4}{*}[22]{} \\
\hline $25-59$ & 10104285 & / & / & gamma & \\
\hline $20-24$ & 790158 & / & / & gamma & \\
\hline $0-19$ & 327225 & / & / & gamma & \\
\hline \multicolumn{6}{|c|}{ People who recovered after being infected } \\
\hline$\geq 60$ & 1463858 & / & l & gamma & \multirow[t]{4}{*}[25,27]{} \\
\hline $25-59$ & 3764975 & / & l & gamma & \\
\hline $20-24$ & 647780 & / & / & gamma & \\
\hline $0-19$ & 1055880 & / & / & gamma & \\
\hline \multicolumn{6}{|l|}{$\begin{array}{l}\text { People who have been infected in the } \\
\text { incubation period }\end{array}$} \\
\hline$\geq 60$ & 3624790 & / & / & gamma & \multirow[t]{2}{*}[25,27]{} \\
\hline $25-59$ & 9322796 & / & / & gamma & \\
\hline $20-24$ & 1604026 & / & I & gamma & \\
\hline $0-19$ & 2614559 & / & l & gamma & \\
\hline \multicolumn{6}{|l|}{ Quarantined people } \\
\hline$\geq 60$ & 0 & / & / & / & \multirow[t]{4}{*}{ / } \\
\hline $25-59$ & 0 & / & / & / & \\
\hline $20-24$ & 0 & / & / & / & \\
\hline $0-19$ & 0 & / & l & / & \\
\hline \multicolumn{6}{|l|}{ Exposed people who have been infected } \\
\hline$\geq 60$ & 2911060 & / & / & gamma & \multirow{4}{*}{$\begin{array}{l}\text { Confirmed cases-quarantined people- } \\
\text { hospitalized people-people recovered after } \\
\text { infection }\end{array}$} \\
\hline $25-59$ & 7487115 & / & l & gamma & \\
\hline $20-24$ & 1288190 & / & / & gamma & \\
\hline $0-19$ & 2099746 & / & / & gamma & \\
\hline \multicolumn{6}{|l|}{ Hospitalized people } \\
\hline$\geq 60$ & 179077 & / & l & gamma & \multirow[t]{4}{*}[28,29]{} \\
\hline $25-59$ & 460578 & / & / & gamma & \\
\hline $20-24$ & 79244 & / & l & gamma & \\
\hline $0-19$ & 129168 & / & / & gamma & \\
\hline
\end{tabular}




\begin{tabular}{|c|c|c|c|c|c|}
\hline Initial number of susceptible people & Value & Range & & Distribution & Source \\
\hline \multicolumn{6}{|l|}{ Hospitalized people with ICU } \\
\hline$\geq 60$ & 23397 & / & / & gamma & \multirow[t]{4}{*}[28,29]{} \\
\hline $25-59$ & 60176 & / & / & gamma & \\
\hline $20-24$ & 10353 & / & / & gamma & \\
\hline $0-19$ & 16876 & / & / & gamma & \\
\hline \multicolumn{6}{|l|}{ The cure and mortality rate/ day } \\
\hline \multicolumn{6}{|l|}{ Natural mortality } \\
\hline$\geq 60$ & $0.0501 \%$ & $0.0476 \%$ & $0.0526 \%$ & beta & \multirow[t]{4}{*}{ [30] } \\
\hline $25-59$ & $0.0032 \%$ & $0.0030 \%$ & $0.0033 \%$ & beta & \\
\hline $20-24$ & $0.0001 \%$ & $0.0001 \%$ & $0.0001 \%$ & beta & \\
\hline $0-19$ & $0.0033 \%$ & $0.0031 \%$ & $0.0035 \%$ & beta & \\
\hline \multicolumn{6}{|l|}{ Out-of-hospital cure rate } \\
\hline$\geq 60$ & $7.88 \%$ & / & / & / & \multirow[t]{4}{*}{ Calculated from out-of-hospital mortality rate } \\
\hline $25-59$ & $10.29 \%$ & / & / & / & \\
\hline $20-24$ & $10.41 \%$ & / & / & / & \\
\hline $0-19$ & $10.41 \%$ & / & / & / & \\
\hline \multicolumn{6}{|l|}{ In-hospital cure rate } \\
\hline$\geq 60$ & $2.67 \%$ & / & / & / & \multirow[t]{4}{*}{ Calculated from in-hospital mortality rate } \\
\hline $25-59$ & $4.57 \%$ & / & / & / & \\
\hline $20-24$ & $4.92 \%$ & / & / & / & \\
\hline $0-19$ & $4.99 \%$ & / & / & / & \\
\hline \multicolumn{6}{|l|}{ Out-of-hospital mortality rate } \\
\hline$\geq 60$ & $3.8101 \%$ & $3.6196 \%$ & $4.0006 \%$ & beta & \multirow[t]{4}{*}{ [39] } \\
\hline $25-59$ & $0.2167 \%$ & $0.2059 \%$ & $0.2276 \%$ & beta & \\
\hline $20-24$ & $0.0050 \%$ & $0.0047 \%$ & $0.0052 \%$ & beta & \\
\hline $0-19$ & $0.0046 \%$ & $0.0044 \%$ & $0.0049 \%$ & beta & \\
\hline \multicolumn{6}{|l|}{ In-hospital mortality rate } \\
\hline$\geq 60$ & $3.1967 \%$ & $3.0368 \%$ & $3.3565 \%$ & beta & \multirow[t]{4}{*}[40]{} \\
\hline $25-59$ & $0.8790 \%$ & $0.8350 \%$ & $0.9229 \%$ & beta & \\
\hline $20-24$ & $0.2855 \%$ & $0.2712 \%$ & $0.2998 \%$ & beta & \\
\hline $0-19$ & $0.1562 \%$ & $0.1484 \%$ & $0.1640 \%$ & beta & \\
\hline \multicolumn{6}{|l|}{$\begin{array}{l}\text { Average rate of unprotected contact infection } \\
\text { per person }\end{array}$} \\
\hline \multicolumn{6}{|l|}{ Number of effective contacts/day } \\
\hline$\geq 60$ & 14.29 & 12.86 & 15.71 & gamma & \multirow[t]{4}{*}{ [31] } \\
\hline $25-59$ & 14.91 & 13.42 & 16.40 & gamma & \\
\hline $20-24$ & 12.68 & 11.41 & 13.95 & gamma & \\
\hline $0-19$ & 12.67 & 11.41 & 13.94 & gamma & \\
\hline $\begin{array}{l}\text { Contact infection rate of people in the } \\
\text { incubation period per people }\end{array}$ & $3.30 \%$ & $2.97 \%$ & $3.63 \%$ & gamma & {$[41]$} \\
\hline $\begin{array}{l}\text { Contact infection rate of exposed people per } \\
\text { people }\end{array}$ & $14.50 \%$ & $13.05 \%$ & $15.95 \%$ & gamma & [42] \\
\hline $\begin{array}{l}\text { Utilization rate of protection measures and } \\
\text { protection rate }\end{array}$ & & & & & \\
\hline
\end{tabular}




\begin{tabular}{|c|c|c|c|c|c|}
\hline Initial number of susceptible people & Value & Range & & Distribution & Source \\
\hline \multicolumn{6}{|l|}{$\begin{array}{l}\text { Usage rate of masks and other protective } \\
\text { measures }\end{array}$} \\
\hline$\geq 60$ & $68.22 \%$ & $64.81 \%$ & $71.63 \%$ & beta & \multirow[t]{4}{*}{ [43] } \\
\hline $25-59$ & $60.02 \%$ & $57.02 \%$ & $63.02 \%$ & beta & \\
\hline $20-24$ & $56.43 \%$ & $53.60 \%$ & $59.25 \%$ & beta & \\
\hline $0-19$ & $56.41 \%$ & $53.59 \%$ & $59.23 \%$ & beta & \\
\hline $\begin{array}{l}\text { Overall protection rate of protective measures } \\
\text { such as masks }\end{array}$ & $98.55 \%$ & $93.62 \%$ & $100.00 \%$ & beta & [44] \\
\hline \multicolumn{6}{|l|}{ Other parameters } \\
\hline \multicolumn{6}{|l|}{ Incubation period $\rightarrow$ exposed period } \\
\hline$\geq 60$ & $18.87 \%$ & $17.92 \%$ & $19.81 \%$ & beta & \multirow[t]{4}{*}{ [32] } \\
\hline $25-59$ & $18.87 \%$ & $17.92 \%$ & $19.81 \%$ & beta & \\
\hline $20-24$ & $18.87 \%$ & $17.92 \%$ & $19.81 \%$ & beta & \\
\hline $0-19$ & $18.87 \%$ & $17.92 \%$ & $19.81 \%$ & beta & \\
\hline Hospital admission rate & $2.10 \%$ & $2.00 \%$ & $2.21 \%$ & beta & {$[25,27]$} \\
\hline The usage rate of ICU & $13.07 \%$ & $12.42 \%$ & $13.72 \%$ & beta & $\begin{array}{l}\text { ICU initial number/total number of } \\
\text { hospitalizations }\end{array}$ \\
\hline The protection rate of vaccine with 1 dose & $52.00 \%$ & $29.50 \%$ & $68.40 \%$ & beta & \multirow[t]{2}{*}{ [45] } \\
\hline The protection rate of vaccine with 2 doses & $95.00 \%$ & $90.30 \%$ & $97.60 \%$ & beta & \\
\hline Number of people vaccinated/ day & 1000000 & 900000 & 1100000 & gamma & [46] \\
\hline The adverse reaction rate of vaccine & $0.00 \%$ & / & / & / & / \\
\hline Willingness to pay threshold & $\$ 63,416.0$ & / & / & / & [37] \\
\hline \multicolumn{6}{|l|}{ Parameters of cost } \\
\hline The cost of hospital & $\$ 15.3$ & $\$ 13.7$ & $\$ 16.8$ & gamma & \multirow[t]{4}{*}[27,36]{} \\
\hline The cost of ICU & $\$ 2,495.3$ & $\$ 2,245.7$ & $\$ 2,744.8$ & gamma & \\
\hline The cost of vaccine/ dose & $\$ 35.0$ & $\$ 31.5$ & $\$ 38.5$ & gamma & \\
\hline $\begin{array}{l}\text { The cost of transportation management and } \\
\text { vaccination }\end{array}$ & $\$ 14.4$ & $\$ 13.0$ & $\$ 15.9$ & gamma & \\
\hline The cost of adverse reaction & $\$ 0.0$ & / & / & / & / \\
\hline Annual discount rate & $3.00 \%$ & $0 \%$ & $5 \%$ & beta & \\
\hline \multicolumn{6}{|l|}{ Parameters of utility } \\
\hline The utility of healthy people & 1 & & & / & / \\
\hline The utility of people recovered & 0.93 & 0.80 & 1.00 & beta & [33] \\
\hline The utility of people in incubation period & 0.96 & 0.92 & 1.00 & beta & [34] \\
\hline The utility of quarantined people & 0.93 & 0.80 & 1.00 & beta & [33] \\
\hline The utility of exposed people & 0.60 & 0.56 & 0.65 & beta & [35] \\
\hline The utility of people in hospital & 0.7 & 0.67 & 0.74 & beta & \multirow[t]{2}{*}{ [27] } \\
\hline The utility of people in ICU & 0.5 & 0.48 & 0.53 & beta & \\
\hline
\end{tabular}

\subsubsection{Demographic parameters}

Because people are generally susceptible to SARS-CoV-2 infection, we set the entire United States population as susceptible and obtained the value of corresponding age groups according to the 2012 Statistical Yearbook of the United States [25]. The number of healthy people receiving the COVID-19 vaccine was converted based on statistical data on the CDC website [26] and according to age groups in this study. The number of people recovered after infection and the number of people infected in the incubation period were calculated based on Kohli's research data combined with the age distribution of the United States population $[25,27]$. The number of exposed people who are susceptible to infection was calculated as the number of confirmed cases minus 
quarantined people minus hospitalized people minus people recovered after infection. Both the total number of people hospitalized and the number of people hospitalized in the intensive care unit (ICU) were derived from statistical data on the CDC website [28, 29].

\subsubsection{Epidemiological parameters}

Because of the 1-day cycle period used in this study, we converted annual and monthly rates in the acquired data into daily rates (including natural mortality rate and in-hospital/out-of-hospital cure/mortality rate). Taking the annual rate as an example, the calculation of the daily rate is based on the following equation:

$$
y=(1+x)^{\frac{1}{365}}-1
$$

where $\mathrm{x}$ is the annual rate and $\mathrm{y}$ is the daily rate.

Natural mortality rate was converted based on statistical data retrieved from the CDC website in 2018 and according to age [30]. Out-of-hospital mortality rate was derived from weekly reports of COVID-19 tracking data on the CDC website. In-hospital mortality rate came from the Morbidity and Mortality Weekly Report (MMWR) on the CDC website. In-hospital and out-of-hospital cure rates were obtained by subtracting the initial data of in-hospital and out-of-hospital mortality rates from 1 and then converted into a daily rate. Because the United States did not take effective quarantine measures in the early stage of the COVID-19 pandemic, we set the number of quarantined people to zero with a quarantine rate of zero. The number of contacts in different age groups of ordinary people was estimated based on the number of contacts in each age group before the outbreak of the pandemic in Wuhan, China [31]. The probability of transferring from incubation period to exposed period was calculated by dividing the cycle period by the duration of the incubation period, and the duration of the incubation period was extracted from Lauer's study [32]. The usage rate of the ICU was calculated by dividing the number of people hospitalized in the ICU by the total number of people hospitalized.

\subsection{Economic Evaluation}

\subsubsection{Health Utility}

The health utility of healthy people was set to one, and the health utility of dead people was set to zero. Because no study has reported the health utility of people recovered after infection with SARS-CoV-2, we collected the data from an investigation of recovered influenza patients in the United States [33]. The health utility of patients in the incubation period was derived from a study on influenza pandemics [34], and the health utility of exposed people came from a study on influenza patients [35]. Furthermore, the health utilities of patients hospitalized and those hospitalized in the ICU were derived from Kohli's research data [27].

\subsubsection{Costs}

Because of a lack of reports on the cost of quarantine for COVID-19 and our assumption of a quarantine rate of zero, we did not take into account the cost of quarantine in this study. The cost of hospital treatment, cost of ICU treatment, cost of vaccine, and cost of transportation management and vaccination were all derived from Kohli's research data and statistical data provided by the United States Centers for Medicare \& Medicaid Services [27, 36]. The cost of adverse reaction treatment and the incidence of adverse reactions were set to zero.

\subsubsection{Willingness-to-pay}

The willingness-to-pay threshold was set to one-time gross domestic product (GDP) per capita of the United States in 2020, and values were derived from data of the International Monetary Fund [37]. Daily discount rate was converted from the annual discount rate of 3\% [27].

\subsubsection{Economic evaluation indicators}

To determine the economy of the intervention measures, we calculated the incremental cost-effectiveness ratio (ICER) and then compared it with the willingness-to-pay threshold. Additionally, we calculated the net benefit compared between different control programs, and the program with the larger net benefit was considered the most economical one (net benefit = benefit - cost). The benefit was defined as direct medical costs saved by a reduction in the number of COVID-19 cases under the vaccination programs considering the cost of vaccine, as compared with the no-vaccination program.

$$
\begin{gathered}
\text { ICER }=\frac{\text { Cost of Intervention B }- \text { Cost of Intervention A }}{\text { QALYs of Intervention B }- \text { QALYs of Intervention A }} \\
\text { Net Benefit }=\text { Benefit }- \text { Cost. }
\end{gathered}
$$

\subsubsection{Uncertainty analysis}

One-way sensitivity analysis, probabilistic sensitivity analysis, and scenario analysis were carried out in this study.

One-way sensitivity analysis: We let 44 parameters including costs fluctuate within a certain range. Based on the fluctuation results, tornado diagrams were drawn to find the major factors influencing the results. 
Probabilistic sensitivity analysis: We assumed that the 44 parameters with uncertainties follow a specific probability distribution. Then, Monte Carlo simulations were carried out 10000 times. Based on the simulation results, a scatterplot and cost-effectiveness acceptability curve were drawn. The economy of the three intervention programs was determined with different willingness-to-pay thresholds. Assuming that the costs follow a Gamma distribution, the utility values have a Beta distribution, the demographic parameters fit a Gamma distribution, and the epidemiological parameters fit a Beta distribution.

Scenario analysis: The analysis was conducted under scenarios assuming that vaccines produced by different manufacturers are administered. Changes in the number of infected people and changes in the ICER were obtained.

All analyses were conducted in Microsoft Excel (Microsoft Corp., Redmond, WA, USA).

\section{Results}

\subsection{Base-case results}

\subsubsection{Net benefit}

Based on a healthcare system perspective, COVID-19 vaccination with priority for either young people or older people results in a lower total cost. Priority vaccination for adults aged 25-59 years (Program 2) saves $\$ 31,664.2$ million in medical costs, and priority vaccination for adults aged over 60 years (Program 3) saves $\$ 30,082.9$ million in medical costs, as compared with no vaccination (Program 1). Because priority vaccination for adults aged $25-59$ years can reduce the number of people infected, this program saves $\$ 1,581.3$ million in medical costs compared with priority vaccination for adults aged over 60 years old. Therefore, in the case of the COVID-19 pandemic, any prioritization strategy of vaccination can save medical costs compared with no vaccine intervention. However, which program is more preferable still needs to be verified by further analysis and research. Details are summarized in Table 2 .

Table 2

Net Benefit

\begin{tabular}{|llllll|}
\hline Intervention program & Treatment cost & Vaccine cost & Total cost & Net benefit & Net benefit \\
\hline Program 1 & 1674032.5 & & 1674032.5 & & \\
\hline Program 2 & 1641177.0 & 1191.3 & 1642368.3 & -31664.2 & \\
\hline Program 3 & 1642652.0 & 1297.6 & 1643949.6 & -30082.9 & 1581.3 \\
\hline Unit: million US dollars & & & & & \\
\hline
\end{tabular}

\subsubsection{ICER}

The cumulative discounted cost per capita and utility per capita simulated in 180 days (calculated based on the mean values of model simulation) are summarized in Table 1. Based on a healthcare system perspective, any vaccination program compared with no intervention results in lower costs and positive economic outcomes, and is thus a very economical measure. Within the simulation period, priority vaccination for adults aged over 60 years costs more than priority vaccination for people aged 25-59 years, while producing better health outcomes. The former program is therefore more economical, when taking onetime GDP per capita of the United States (2020) as the willingness-to-pay threshold. Considering the two vaccination strategies, priority vaccination for adults aged over 60 years gains 0.001 QALYs per capita while costing $\$ 4.7$ per capita more than priority vaccination for adults aged $25-59$ years in the simulation period, with an incremental cost-effectiveness ratio (ICER) of $\$ 4,829.3 /$ QALY. Because the mortality rate of older people is higher than that of young people, the increase in QALYs is mostly due to a reduction in the infections of older people, which prevents the death of older people. In general, all vaccination strategies require additional expenditure for the cost of vaccines compared with no vaccination. However, vaccination still shows an absolute advantage over no vaccination with regard to the strong infectivity of COVID-19 and the high cost of treatment. Details are provided in Table 3.

Table 3

Cost-effectiveness analysis results

\begin{tabular}{|lllll|}
\hline $\begin{array}{l}\text { Intervention } \\
\text { program }\end{array}$ & $\begin{array}{l}\text { Cost per } \\
\text { capita } \\
\text { (dollars) }\end{array}$ & $\begin{array}{l}\text { Utility per } \\
\text { capita } \\
\text { (QALY) }\end{array}$ & $\begin{array}{l}\text { Incremental cost-effectiveness ratio } \\
\text { (\$/QALY) }\end{array}$ & $\begin{array}{l}\text { Incremental cost-effectiveness ratio } \\
\text { (\$/QALY) }\end{array}$ \\
\hline Program 1 & 4989.4 & 0.4112 & & \\
\hline Program 2 & 4895.1 & 0.4128 & -57655.5 & 4829.3 \\
\hline Program 3 & 4899.8 & 0.4138 & -34316.8 & \\
\hline
\end{tabular}

\subsection{Sensitivity analysis}

\subsubsection{One-way sensitivity analysis}


Figure $2(a-c)$ shows three pairs of contrastive programs (Program 2 vs. Program 1; Program 3 vs. Program 1; Program 3 vs. Program 2 ). Details of parameter fluctuations are available in Table 1. For the one-way sensitivity analysis, the five most influential factors are selected and included in tornado diagrams. The results show that although parameter variation affects ICER to a certain degree, the economic results mentioned above are still absolutely robust, and the superior program still holds an absolute dominant position. That is, the two vaccination programs are absolutely superior to no vaccine intervention. Furthermore, priority vaccination for people aged over 60 years vs. 25-59 years old is still absolutely economical, when taking one-time GDP per capita of the United States as the willingness-to-pay threshold. However, Fig. $2(a, b)$ shows that the protection rate of the vaccine is still a major factor influencing the economic results. Although there is currently evidence for the vaccine's protection rate, its protection period still needs to be corroborated by stronger evidence to verify the economy of the vaccine.

\subsubsection{Probabilistic sensitivity analysis}

The distribution of parameters used for probabilistic sensitivity analysis is provided in Table 1. The results (Fig. 2(e)) show that there is no uncertainty in the economic results of vaccination compared with no intervention. All vaccination programs analyzed in this study are advantageous over no vaccination. Priority vaccination for adults aged over 60 years vs. 25-59 years is more economical in the case of taking one-time GDP per capita of the United States as the willingness-to-pay threshold. Furthermore, the cost-effectiveness acceptability curve (Fig. 2(d)) shows that vaccination is absolutely acceptable compared with no vaccination. Furthermore, the $50 \%$ acceptable probability of giving priority to vaccinating adults aged over 60 years is less than the one-time GDP per capita of the United States.

\subsubsection{Scenario analysis}

People in the United States have mostly received the Pfizer vaccine[22], namely BNT162b2. Therefore, the scenario analysis is performed to explore changes in the number of infected people and ICER after receiving vaccines from different manufacturers. The specific scenarios are as follows:

Scenario 1: The AZD1222 vaccine produced by AstraZeneca is administered. The adverse reactions to the vaccine are mainly fatigue and headache. Other common adverse reactions that have been reported include myalgia and discomfort. A small proportion of recipients have Grade 3 systemic adverse reactions such as chills or fever.

Scenario 2: The Ad26.COV2.S vaccine produced by Johnson \& Johnson is administered. The most common adverse reactions are pain at the injection site, headache, fatigue, myalgia, and nausea, most of which are mild or moderate.

Scenario 3: The mRNA-1273 vaccine jointly developed by the National Institute of Allergy and Infectious Diseases (United states National Institute of Health) and Moderna is administered. The most common adverse reactions are pain at the injection site, fatigue, headache, and fever, mostly at Grade 1 and 2.

Scenario 4: The NVX-CoV-2373 vaccine manufactured by Novavax is administered. The incidence of serious adverse reactions is low.

Specific details of the vaccines are summarized in Table 4. Because the adverse reactions are mostly mild, the cost of treatment for adverse drug reactions in each scenario was not considered in the scenario analysis. Because of a lack of research related to the protection rate of a single vaccine dose for some vaccines, the single dose protection rate was set to $52 \%$, which is the protection rate of a single dose of the Pfizer vaccine.

Table 4

Data of scenario analysis

\begin{tabular}{|c|c|c|c|c|c|c|}
\hline Manufacturer & Vaccine & Type & $\begin{array}{l}\text { Price/two } \\
\text { doses }\end{array}$ & $\begin{array}{l}\text { Protection rate } \\
\text { of one-dose } \\
\text { vaccine }\end{array}$ & $\begin{array}{l}\text { Protection rate } \\
\text { of two-dose } \\
\text { vaccine }\end{array}$ & Source \\
\hline AstraZeneca & AZD1222 & $\begin{array}{l}\text { Adenovirus } \\
\text { vector } \\
\text { vaccine }\end{array}$ & $\$ 8.0$ & $64.10 \%$ & $70.40 \%$ & [47] \\
\hline Johnson \& Johnson & Ad26.COV2.S & $\begin{array}{l}\text { Adenovirus } \\
\text { vector } \\
\text { vaccine }\end{array}$ & $\$ 20.0$ & / & $67.00 \%$ & {$[48]$} \\
\hline $\begin{array}{l}\text { National Institute of Allergy and Infectious Diseases } \\
\text { (United states National Institute of Health) and Moderna }\end{array}$ & mRNA-1273 & $\begin{array}{l}\text { mRNA nucleic } \\
\text { acid vaccine }\end{array}$ & $\$ 30.0$ & / & $94.10 \%$ & [49] \\
\hline Novavax & $\begin{array}{l}\text { NVX-CoV- } \\
2373\end{array}$ & $\begin{array}{l}\text { Recombinant } \\
\text { protein } \\
\text { vaccine }\end{array}$ & $\$ 32.0$ & / & $89.30 \%$ & {$[50]$} \\
\hline
\end{tabular}

Vaccination achieves a certain protective effect irrespective of the vaccines (Fig. 3). However, it is assumed that the virus will not mutate; the immigration and emigration of the population are not considered, and there will be a certain period of immunity after infection. Therefore, the number of newly infected people will decrease gradually after reaching a peak. Although the assumptions are necessary and do not have a large influence on the economic results of the model, they do have great limitations. Nonetheless, we have to make these assumptions to explore the rational use of limited resources.

The results of scenario analysis are summarized in Tables 5 and 6. Despite the different types of vaccines administered, the economic results are relatively stable with the one-time GDP per capita of the United States in 2020 as the willingness-to-pay threshold. Vaccination is more economical than no vaccination, 
while priority vaccination for adults aged over 60 years is more economical than priority vaccination for adults aged 25-59 years. Table 6 compares the results between vaccines. BNT162b2 (Pfizer) is significantly superior to the other vaccines without considering the cost of treatment for adverse reactions.

Table 5

Results of scenario analysis

\begin{tabular}{|llll|}
\hline Scenario & ICER1 (\$/QALY) & ICER2 (\$/QALY) & ICER3 (\$/QALY) \\
\hline AZD1222 & -55899.89 & -32049.27 & -7831.54 \\
\hline Ad26.COV2.S & -49192.73 & -30592.18 & -7133.52 \\
\hline mRNA-1273 & -58200.73 & -34822.27 & 4172.20 \\
NVX-CoV-2373 & -56414.74 & -35029.09 & -1646.42 \\
\hline ICER1: priority is given to vaccination for people aged 25-59 years vs. no vaccination \\
ICER2: priority is given to vaccination for people aged over 60 years vs. no vaccination \\
ICER3: priority is given to vaccination for people aged over 60 years vs. 25-59 years \\
\hline
\end{tabular}

Table 6

Results of scenario analysis

\begin{tabular}{|lll|}
\hline Vaccine & Average Cost/\$ & Average QALY \\
\hline BNT162b2 & 4899.8 & 0.4138 \\
\hline mRNA-1273 & 4917.1 & 0.4133 \\
\hline NVX-CoV-2373 & 5000.2 & 0.4113 \\
\hline AZD1222 & 5144.7 & 0.4079 \\
\hline Ad26.COV2.S & 5188.4 & 0.4065 \\
\hline
\end{tabular}

\section{Discussion}

\subsection{Main findings}

This study presents two main findings. First, there is a great possibility that vaccination is economical even without considering the prioritization issue. Because COVID-19 is highly infectious with a relatively high cost of treatment, vaccination is very likely to save considerable medical costs even taking into account the cost of the vaccine. Second, priority vaccination for adults over 25-59 years old reduces the number of infections and thereby saves money. Priority vaccination for adults aged over 60 years reduces the population's mortality rate and thereby achieves better health outcomes, which is more economical than priority vaccination for other age groups. These findings corroborate the views of most people and are in line with the WHO and the United States Food and Drug Administration's understanding of COVID-19 vaccines. Therefore, from the perspective of the public budget, priority should be given to vaccinating adults over 25-59 years old; but from the perspective of health value, priority should be given to vaccinating adults over 60 years old.

In the United States, the incidence of COVID-19 cases can be markedly reduced by daily administration of $\sim 1$ million doses of the Pfizer vaccine. Priority vaccination for adults aged $25-59$ years and over 60 years can reduce $\$ 31,664.2$ million and $\$ 30,082.9$ million in medical costs, respectively, compared with no vaccine intervention. The ICERs for priority vaccination for adults aged $25-59$ years and over 60 years are $-\$ 57,655.5 / Q A L Y$ and $-\$ 34,316.8 / Q A L Y$, respectively, both of which are less than zero. The results of sensitivity analysis show that irrespective of the vaccine type and prioritization of the vaccination program selected, the vaccine group has an absolute advantage over the control group, and the results are extremely stable. Large-scale vaccination is undoubtedly a wise move owing to the high cost of treatment for COVID-19. Although the cost of the vaccine is not low, the necessary treatment approaches involving the ICU and ventilators require high costs. Meanwhile, vaccine intervention is not only effective for the interveners per se, but also provides indirect protection and achieves greater health benefits than expected.

Comparing the vaccination programs with different prioritization orders, priority vaccination for adults aged over 60 years achieves the best health outcomes. The ICER of priority vaccination for adults aged over 60 years vs. $25-59$ years is $\$ 4,829.3 /$ QALY. Priority vaccination for adults aged over 60 years is therefore more effective and economical when taking one-time GDP per capita of the United States in 2020 as the willingness-to-pay threshold. Priority vaccination for adults aged 25-59 years can indeed reduce the number of new COVID-19 cases; however, the mortality rate is highest in adults aged over 60 years who have been infected with the disease; prioritizing this age group for vaccination can reduce the number of deaths and achieve greater health benefits. This conclusion is also extremely stable according to the results of sensitivity analysis.

Here, scenario analysis reveals that vaccination can achieve more health outcomes than no intervention. Furthermore, all the other four COVID-19 vaccines included in the scenario analysis are significantly economical with one-time GDP per capita of the United States as the willingness-to-pay threshold, among which priority vaccination for adults aged over 60 years is the best program. Comparing different vaccines reveals that the BNT162b2 vaccine has an absolute advantage.

\subsection{Strengths and limitations}


Although the United States has now launched universal vaccinations and have applied products from multiple brands which against our assumption, it is difficult for the complexity of reality to be included in the model. With that being said, this research is not meaningless given the fact that it remains difficult to build reasonable and effective models with lack of parameter inputs. We took United States as our study setting because it is the only country with the most complete parameters till now. Since many countries have launched the plan of universal vaccinations but facing the same allocation problems as the United States. As well as other low-income and middle-income countries who do not have enough vaccine resources. We hope the study flow could provide useful reference to discuss the economic value of different vaccination strategies in other countries.

Additionally, the development of acute infectious diseases is often difficult to accurately estimate. Consequently, very few studies have been carried out and published on the economic evaluation of COVID-19 vaccines, and most studies have only estimated the development of the COVID-19 pandemic. The several economic evaluations of COVID-19 vaccines that have been published so far [20,27] mainly focus on evaluating the economy of COVID-19 vaccination without comparing the economy of vaccination strategies with different prioritization orders. This is the first study to evaluate the economic value of the prioritization strategies of COVID-19 vaccination by age groups.

To the best of our knowledge, there have been no studies comprehensively considering and comparing multiple vaccination programs and exploring the economy of different vaccines, as implemented in this study. This study is carried out based on a relatively comprehensive consideration in the scope permitted by actual conditions. We take into account the spread between people and the cross-protection by vaccines and repeatedly weigh clinically important parameters such as vaccine protection rate and treatment cost. We not only draw the conclusion that the vaccine intervention program is absolutely superior to no intervention but also compare the economy of different vaccine intervention programs. The model and results provide a reference of age prioritization for vaccination in most countries that have already carried out universal vaccination or other countries who will have a chance to conduct largescale vaccination in the future.

This study is not without limitations. First, taking into account the disease characteristics, the simulation period of the study was set to 180 days. Although it cannot completely simulate that all people in the United States are vaccinated, we still obtained the results of vaccine economy and the appropriate vaccination program within the priority simulation period. Second, the mutation of the virus is inevitable yet difficult to estimate, and we had to ignore virus mutation. Third, whether the adverse reactions of the vaccines are mostly mild as stated in the literature is still to be discussed or verified by real-world reports. Meanwhile, the sequelae of people recovered also lack strong reports to confirm, and we had to use relevant research reports on similar respiratory diseases as influenza as an alternative. Fourth, we did not consider the cost associated with diagnostic monitoring. Although these assumptions and substitutions are necessary, they could affect the accuracy of the prediction results to a certain extent.

\section{Conclusion}

Currently, COVID-19 vaccines have been on the market for a short time and the population vaccination rate is generally low. Many low-income countries have not started vaccination. Therefore, both the effectiveness and safety of the vaccines lack strong real-world evidence for corroboration. Nonetheless, vaccines are undoubtedly an important part in many of the prevention strategies for COVID-19. So far, Israel is the only country that has achieved universal vaccination, whereas most countries in the world are still far from achieving universal vaccination[38].

This study indicates that COVID-19 vaccination in the United States provides significant health and economic benefits. Despite the high price of the vaccine, compared with the high cost of treatment, vaccination is still an extremely cost-effective choice based on a healthcare perspective. Age grouping is indeed a desirable option of prioritization for vaccination. However, whether there is a greater risk of infection for some occupations, such as medical workers, and whether these occupations should be considered for priority vaccination remain to be studied.

\section{Abbreviations}

QALY

Quality-adjusted life year; COVID-19:coronavirus disease 2019; R0:mean reproductive rate; ICER:incremental cost-effectiveness ratio; WHO:World Health Organization; CDC:the United States Centers for Disease Control and Prevention; ICU:intensive care unit; GDP:gross domestic product

\section{Declarations}

\section{Ethics approval and consent to participate}

Ethics approval was not applicable because the primary data were extracted from the published literature.

\section{Consent for publication}

Not applicable.

\section{Availability of data and materials}

We used publicly available data sources. However, codes and results are available from the authors upon reasonable request. 


\section{Competing interests}

The authors declare that the research was conducted in the absence of any commercial or financial relationships that could be construed as a potential conflict of interest.

\section{Funding}

This study was supported by the National Natural Science Foundation of China (Grant No.: 71603278) provided to WT.

\section{Authors' contributions}

WT designed the study. DZ built and validated the model. Data collection and parameterization were completed by DZ, TS, ZL, XP, and XZ. DZ and TS conducted the analysis. DZ, TS, ZL and CZ wrote the initial draft of the manuscript. All co-authors critically revised the manuscript and approved the final version.

\section{Acknowledgement}

We thank Lucy McClellan, MIA, from Liwen Bianji (Edanz) (www.liwenbianji.cn/), for editing the English text of a draft of this manuscript.

\section{References}

1. Foy BH, Wahl B, Mehta K, Shet A, Menon GI, Britto C: Comparing COVID-19 vaccine allocation strategies in India: A mathematical modelling study. INT J INFECT DIS. 2021; 103:431-438.

2. WHO. WHO Coronavirus Disease (COVID-19) Dashboard; 2021. https://covid19.who.int/. Accessed 20 April 2021

3. Chen H, Guo J, Wang C, Luo F, Yu X, Zhang W, Li J, Zhao D, Xu D, Gong Q et al: Clinical characteristics and intrauterine vertical transmission potential of COVID-19 infection in nine pregnant women: a retrospective review of medical records. LANCET. 2020; 395(10226):809-815.

4. Khan M, Adil SF, Alkhathlan HZ, Tahir MN, Saif S, Khan M, Khan ST: COVID-19: A Global Challenge with Old History, Epidemiology and Progress So Far. MOLECULES. 2020; 26(1).

5. Backer JA, Klinkenberg D, Wallinga J: Incubation period of 2019 novel coronavirus (2019-nCoV) infections among travellers from Wuhan, China, 20-28 January 2020. Euro Surveill. 2020; 25(5).

6. Sidney Daily News. COVID-19 by numbers; 2021. https://www.sidneydailynews.com/news/174005/covid-19-by-the-numbers. Accessed 21 March 2021

7. de Wit E, van Doremalen N, Falzarano D, Munster VJ: SARS and MERS: recent insights into emerging coronaviruses. NAT REV MICROBIOL. 2016; 14(8):523-534.

8. Wu YC, Chen CS, Chan YJ: The outbreak of COVID-19: An overview. J CHIN MED ASSOC. 2020; 83(3):217-220.

9. Chen WS, Hou J: How much does COVID-19 cost, from nucleic acid testing to entering ICU, and from mild to critical case treatment? China Economic Weekly. 2020(07):54-57.

10. Jin Y, Cai L, Cheng Z, Cheng H, Deng T, Fan Y, Fang C, Huang D, Huang L, Huang Q et al: A rapid advice guideline for the diagnosis and treatment of 2019 novel coronavirus (2019-nCoV) infected pneumonia (standard version). Military Medical Research. 2020; 7(1).

11. WHO. Clinical Management of Covid-19; 2020. https://www.who.int/publications-detail-redirect/clinical-management-of-covid-19. Accessed 28 Jan 2021

12. National Health Commission of the People's Republic of China. New coronavirus pneumonia diagnosis and treatment plan(Trial eighth edition); 2020. http://www.nhc.gov.cn/cms-search/downFiles/a449a3e2e2c94d9a856d5faea2ff0f94.pdf. Accessed 28 Jan 2021

13. Zhu F, Guan X, Li Y, Huang J, Jiang T, Hou L, Li J, Yang B, Wang L, Wang W et al: Immunogenicity and safety of a recombinant adenovirus type-5-vectored COVID-19 vaccine in healthy adults aged 18 years or older: a randomised, double-blind, placebo-controlled, phase 2 trial. The Lancet (British edition). 2020; 396(10249):479-488.

14. Fang CD, Zhang YH, Wu HF, Ye HY: Research progress in COVID-19 vaccine. China Pharmacist. 2020; 23(9):1826-1830.

15. Xinhua. World needs safe, effective vaccines available for all, says UN ECOSOC; 2021. http://xinhuanet.com/english/2021-04/21/c_139894624.htm. Accessed 23 April 2021

16. Abecassis A: Five priorities for universal COVID-19 vaccination. LANCET. 2021; 398(10297):285-286.

17. The LID: The rocky road to universal COVID-19 vaccination. LANCET INFECT DIS. 2021; 21(6):743.

18. The White House. Fact Sheet: President Biden to Announce All Americans to be Eligible for Vaccinations by May 1, Puts the Nation on a Path to Get Closer to Normal by July 4th; 2021. https://www.whitehouse.gov/briefing-room/statements-releases/2021/03/11/fact-sheet-president-biden-to-announce-allamericans-to-be-eligible-for-vaccinations-by-may-1-puts-the-nation-on-a-path-to-get-closer-to-normal-by-july-4th/. Accessed 21 March 2021

19. Kaiser Health News. Some Said the Vaccine Rollout Would Be a 'Nightmare.' They Were Right; 2020. https://www.thefiscaltimes.com/2020/12/28/SomeSaid-Vaccine-Rollout-Would-Be-Nightmare-They-Were-Right. Accessed March 212021

20. Bubar KM, Reinholt K, Kissler SM, Lipsitch M, Cobey S, Grad YH, Larremore DB: Model-informed COVID-19 vaccine prioritization strategies by age and serostatus. SCIENCE. 2021; 371(6532):916-921. 
21. Schmidt H, Pathak P, Sonmez T, Unver MU: Covid-19: how to prioritize worse-off populations in allocating safe and effective vaccines. BMJ. 2020; 371:m3795.

22. Centers for Disease Control and Prevention. COVID-19 Vaccinations in the United States; https://covid.cdc.gov/covid-data-tracker/\#vaccinations. Accessed 21 March 2021

23. Ripperger TJ, Uhrlaub JL, Watanabe M, Wong R, Castaneda Y, Pizzato HA, Thompson MR, Bradshaw C, Weinkauf CC, Bime C et al: Orthogonal SARS-CoV-2 Serological Assays Enable Surveillance of Low-Prevalence Communities and Reveal Durable Humoral Immunity. IMMUNITY. 2020; 53(5):925-933.

24. McMurry R, Lenehan P, Awasthi S, Silvert E, Puranik A, Pawlowski C, Venkatakrishnan AJ, Anand P, Agarwal V, O Textquoteright Horo JC et al: Real-time analysis of a mass vaccination effort via an Artificial Intelligence platform confirms the safety of FDA-authorized COVID-19 vaccines. medRxiv. 2021.

25. Statistical abstract of the United States. Washington: U.S. G.P.O.; 2012.

26. Centers for Disease Control and Prevention. COVID-19 Vaccinations in the United States; 2021. https://covid.cdc.gov/covid-data-tracker/\#vaccinations. Accessed 5 March 2021

27. Kohli M, Maschio M, Becker D, Weinstein MC: The potential public health and economic value of a hypothetical COVID-19 vaccine in the United States: Use of cost-effectiveness modeling to inform vaccination prioritization. VACCINE. 2021; 39(7):1157-1164.

28. U.S. Department of Health \& Human Services. COVID-19 Reported Patient Impact and Hospital Capacity by Facility; 2021. https://healthdata.gov/dataset/covid-19-reported-patient-impact-and-hospital-capacity-facility. Accessed 5 March 2021

29. Centers for Disease Control and Prevention. Estimated Disease Burden of COVID-19; 2021. https://www.cdc.gov/coronavirus/2019-ncov/casesupdates/burden.html. Accessed 5 March 2021

30. Centers for Disease Control and Prevention. Mortality in the United States, 2018; 2020. https://www.cdc.gov/nchs/products/databriefs/db355.htm. Accessed 5 March 2021

31. Zhang J, Litvinova M, Liang Y, Wang Y, Wang W, Zhao S, Wu Q, Merler S, Viboud C, Vespignani A et al: Changes in contact patterns shape the dynamics of the COVID-19 outbreak in China. SCIENCE. 2020; 368(6498):1481-1486.

32. Lauer SA, Grantz KH, Bi Q, Jones FK, Zheng Q, Meredith HR, Azman AS, Reich NG, Lessler J: The Incubation Period of Coronavirus Disease 2019 (COVID19) From Publicly Reported Confirmed Cases: Estimation and Application. ANN INTERN MED. 2020; 172(9):577-582.

33. Perlroth DJ, Glass RJ, Davey VJ, Cannon D, Garber AM, Owens DK: Health outcomes and costs of community mitigation strategies for an influenza pandemic in the United States. CLIN INFECT DIS. 2010; 50(2):165-174.

34. Kamal MA, Smith PF, Chaiyakunapruk N, Wu D, Pratoomsoot C, Lee K, Chong HY, Nelson RE, Nieforth K, Dall G et al: Interdisciplinary pharmacometrics linking oseltamivir pharmacology, influenza epidemiology and health economics to inform antiviral use in pandemics. Br J Clin Pharmacol. 2017; 83(7):1580-1594

35. Lee BY, McGlone SM, Bailey RR, Wiringa AE, Zimmer SM, Smith KJ, Zimmerman RK: To test or to treat? An analysis of influenza testing and antiviral treatment strategies using economic computer modeling. PLOS ONE. 2010; 5(6):e11284.

36. U.S. Centers for Medicare \& Medicaid. Medicare COVID-19 Vaccine Shot Payment; https://www.cms.gov/medicare/covid-19/medicare-covid-19-vaccineshot-payment. Accessed 5 March 2021

37. International Monetary Fund. World Economic Outlook Database; https://www.imf.org/en/Publications/WEO/weo-database/2021/April/weo-report? $C=111$,\&s=NGDP_R,NGDP_RPCH,NGDP,NGDPD,PPPGDP,NGDP_D,NGDPRPC,NGDPRPPPPC,NGDPPC,NGDPDPC,PPPPC,NGAP_NPGDP,PPPSH,PPPEX,NID_A Accessed 7 April 2021

38. Mallapaty S: Vaccines are curbing COVID: Data from Israel show drop in infections. NATURE. 2021; 590(7845):197.

39. Centers for Disease Control and Prevention. COVID-19 Weekly Cases and Deaths per 100,000 Population by Age, Race/Ethnicity, and Sex; https://covid.cdc.gov/covid-data-tracker/\#demographicsovertime. Accessed 5 March 2021

40. Centers for Disease Control and Prevention. Morbidity and Mortality Weekly Report (MMWR); https://www.cdc.gov/mmwr/volumes/69/wr/mm6924e2.htm? Accessed 5 March 2021

41. Liu T, Liang W, Zhong H, He J, Chen Z, He G, Song T, Chen S, Wang P, Li J et al: Risk factors associated with COVID-19 infection: a retrospective cohort study based on contacts tracing. Emerg Microbes Infect. 2020; 9(1):1546-1553.

42. Burke RM, Balter S, Barnes E, Barry V, Bartlett K, Beer KD, Benowitz I, Biggs HM, Bruce H, Bryant-Genevier J et al: Enhanced contact investigations for nine early travel-related cases of SARS-CoV-2 in the United States. PLOS ONE. 2020; 15(9):e238342.

43. Rader B, White LF, Burns MR, Chen J, Brilliant J, Cohen J, Shaman J, Brilliant L, Kraemer M, Hawkins JB et al: Mask-wearing and control of SARS-CoV-2 transmission in the USA: a cross-sectional study. Lancet Digit Health. 2021; 3(3):e148-e157.

44. Bundgaard H, Bundgaard JS, Raaschou-Pedersen D, von Buchwald C, Todsen T, Norsk JB, Pries-Heje MM, Vissing CR, Nielsen PB, Winslow UC et al: Effectiveness of Adding a Mask Recommendation to Other Public Health Measures to Prevent SARS-CoV-2 Infection in Danish Mask Wearers: A Randomized Controlled Trial. ANN INTERN MED. 2021; 174(3):335-343.

45. Vergnes JN: Safety and Efficacy of the BNT162b2 mRNA Covid-19 Vaccine. N Engl J Med. 2021; 384(11).

46. The White House. Remarks by President Biden on the 100 Million Shot Goal; https://www.whitehouse.gov/briefing-room/speechesremarks/2021/03/18/remarks-by-president-biden-on-the-100-million-shot-goal/. Accessed 5 March 2021

47. Voysey M, Clemens S, Madhi SA, Weckx LY, Folegatti PM, Aley PK, Angus B, Baillie VL, Barnabas SL, Bhorat QE et al: Safety and efficacy of the ChAdOx1 nCoV-19 vaccine (AZD1222) against SARS-CoV-2: an interim analysis of four randomised controlled trials in Brazil, South Africa, and the UK. LANCET. 2021; 397(10269):99-111. 
48. European Medicines Agency. COVID-19 Vaccine Janssen; 2021. https://www.ema.europa.eu/en/medicines/human/EPAR/covid-19-vaccine-janssen. Accessed 20 April 2021

49. Baden LR, El SH, Essink B, Kotloff K, Frey S, Novak R, Diemert D, Spector SA, Rouphael N, Creech CB et al: Efficacy and Safety of the mRNA-1273 SARSCoV-2 Vaccine. N Engl J Med. 2021; 384(5):403-416.

50. Novavax. Novavax COVID-19 Vaccine Demonstrates 89.3\% Efficacy in UK Phase 3 Trial; 2021. https://ir.novavax.com/news-releases/news-releasedetails/novavax-covid-19-vaccine-demonstrates-893-efficacy-uk-phase-3. Accessed 20 April 2021

\section{Figures}

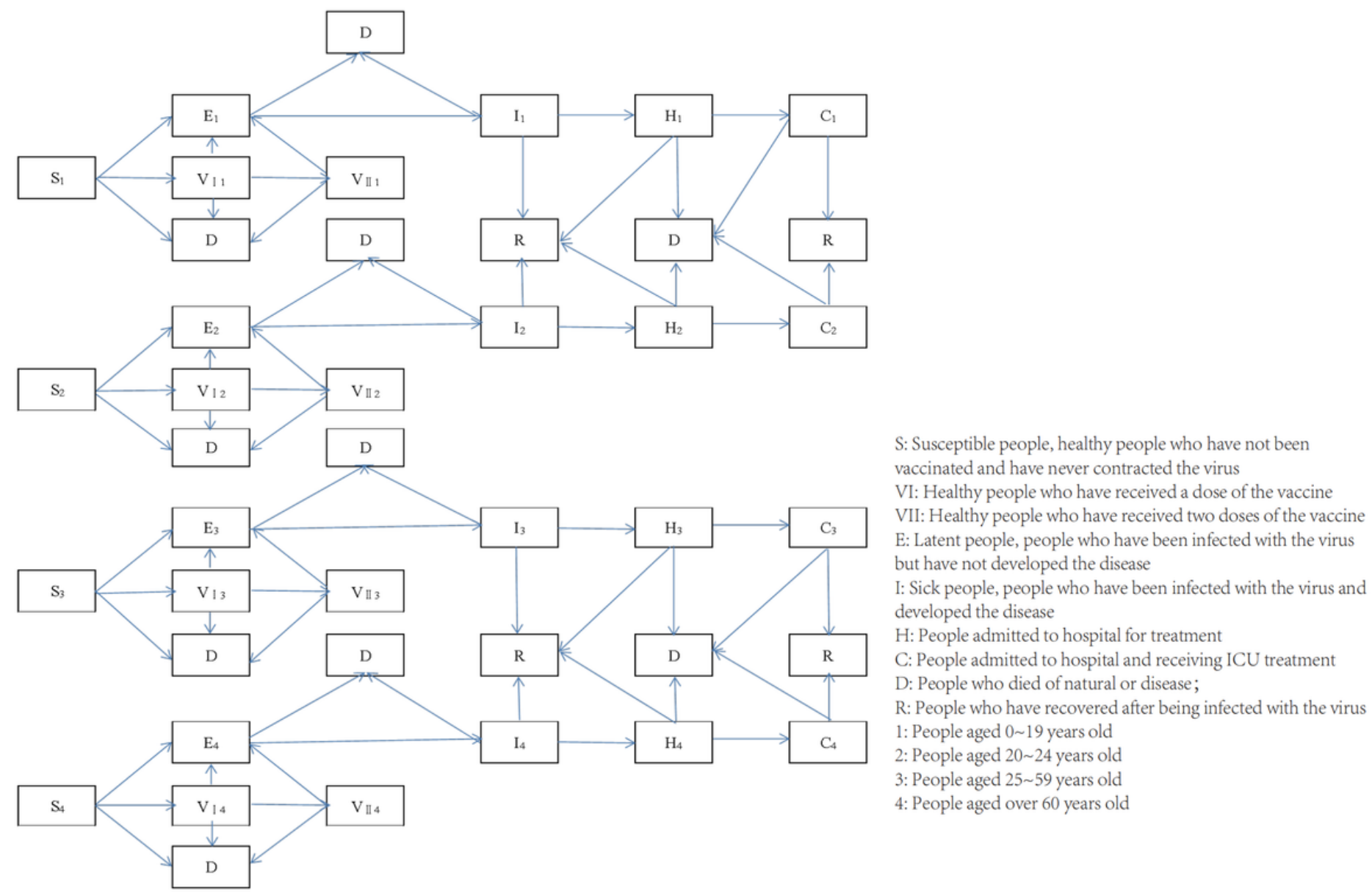

Figure 1

Model Structure 


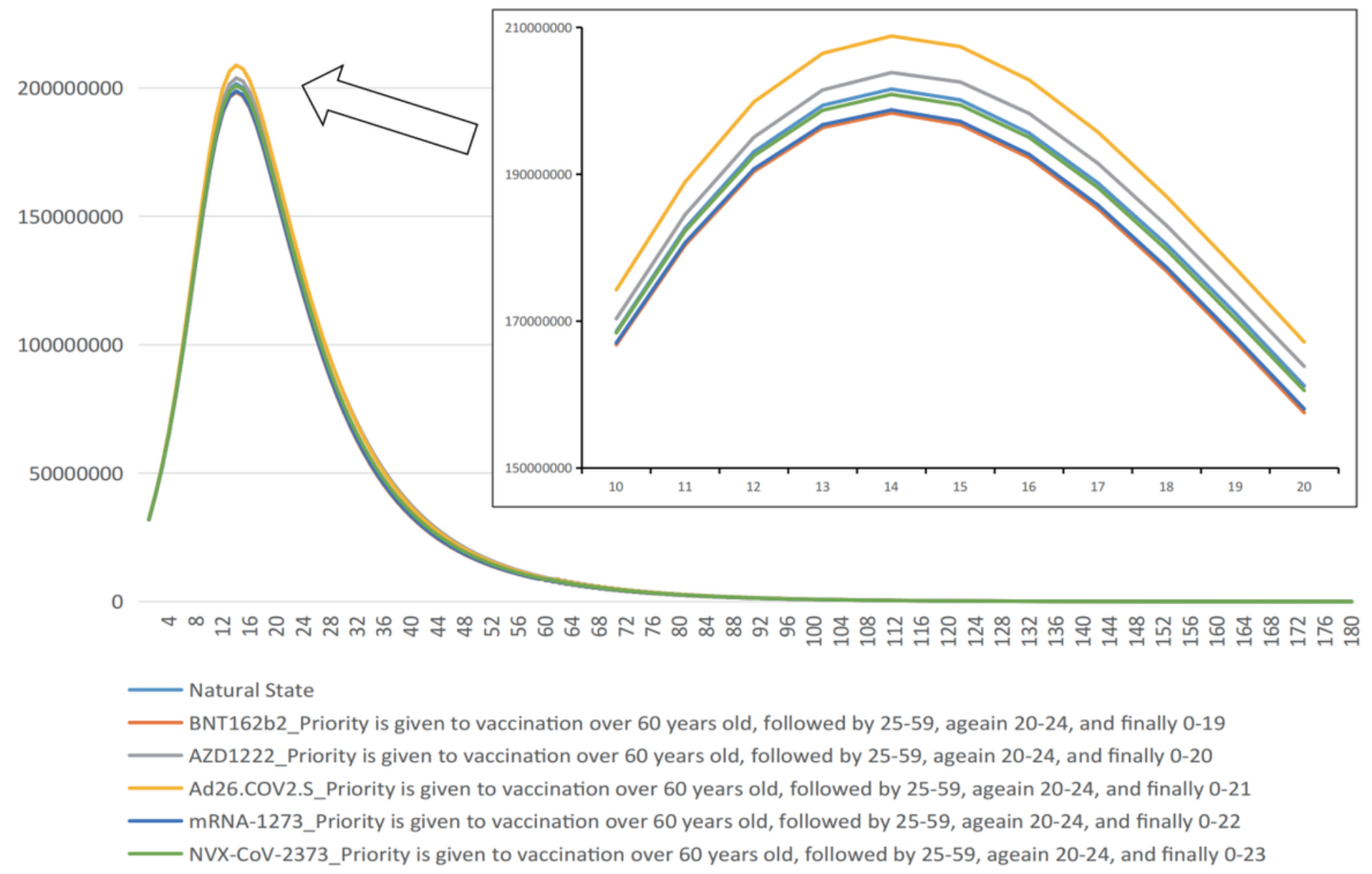

Figure 2

Results of Sensitivity Analysis 

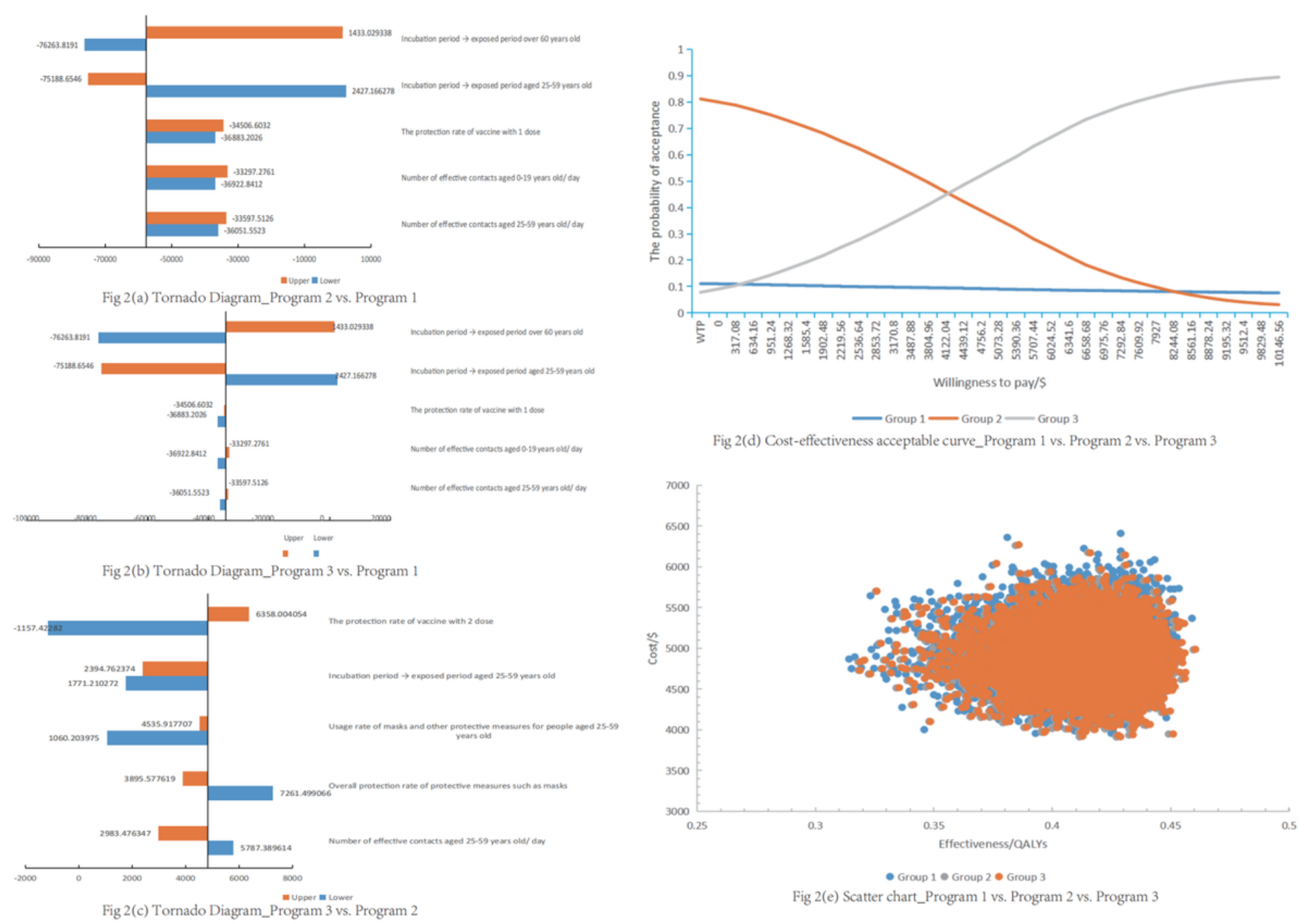

Fig 2(d) Cost-effectiveness acceptable curve_Program 1 vs. Program 2 vs. Program 3

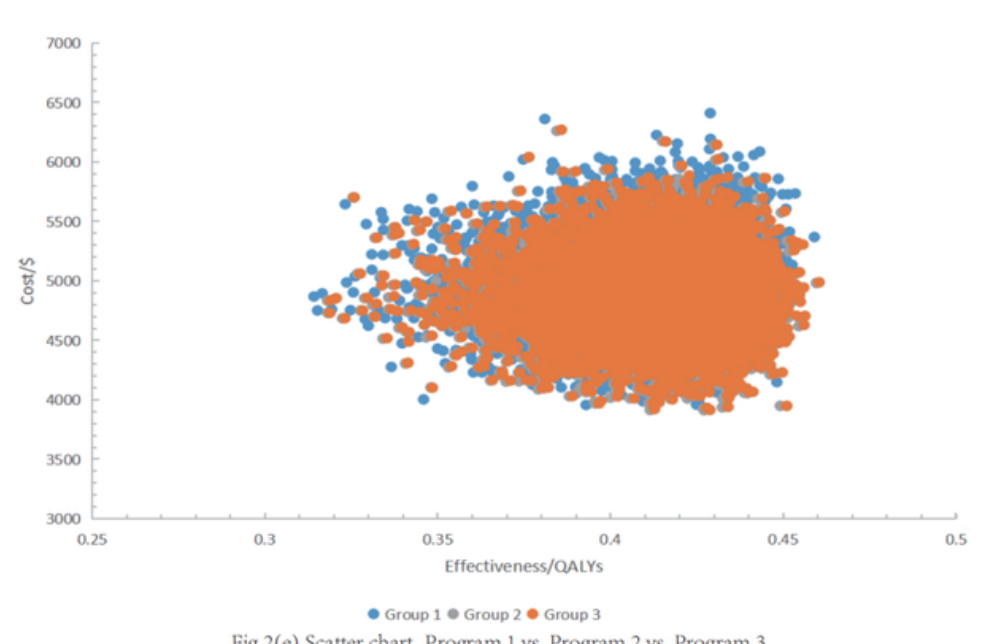

Fig 2(e) Scatter chart_Program 1 vs. Program 2 vs. Program 3

\section{Figure 3}

Changes in the number of newly infected people under different vaccination programs in different scenario

\section{Supplementary Files}

This is a list of supplementary files associated with this preprint. Click to download.

- COVID19model.zip 